\title{
Investigating an active cooling system powered by a thermoelectric generator
}

\author{
Pietro Tosato, Maurizio Rossi, and Davide Brunelli \\ Industrial Engineering, University of Trento \\ via Sommarive 9, 38123 Trento, Italy
}

\begin{abstract}
The diffusion of powerful microprocessors, even in embedded processing, justifies the need for more efficient heat dissipation. Even though the implementation of active cooling is often easy, in this study, we show how to lower the temperature of a general-purpose microprocessor with energy harvesting techniques. We present a joint thermal and electrical analysis of a thermoelectric-powered active cooler and we demonstrate that it is possible to decrease the temperature some Celsius degrees with respect to a regular passive cooler using the same energy dissipated by the processor under the heat-sink.
\end{abstract}

Keywords: Thermoelectric generator, Energy harvesting, Energy Neutral system, Thermoelectric cooling

\section{Introduction}

Energy harvesting is nowadays a consolidated process to convert, and store electrical energy for consumer and embedded electronics, from external environmental sources (e.g., solar power [2] [1], wind energy [14] [11], kinetic energy, bacteria $[19,4]$ or electromagnetic coupling [13]). Thermoelectric energy harvesting is a well-known example of such techniques, especially in wearable applications [3], taking advantage of the difference between human body and air; and in smart sensors deployed in industrial plants, exploiting the wasted heat at high temperatures from industrial thermal processes [18].

There are many applications of Thermoelectric Generators (TEG) in literature, most of them are focused on applications where high power is involved, like in automotive, aircraft and spacecraft, combining heat and solar for thermoelectric power generators [6].

With the development of low-power electronics and new battery generations [12], TEGs were used also in the market of smart sensing electronics. One of the first contributions on energy harvesting from the microprocessor wasted heat can be found back in 2008 from Zhou et al. [22], who presented the problem of heat spreading from the microprocessor chip and the difference between benchmarks and load type on the harvested power.

A more recent investigation is provided in [16], where an energy neutral cooling system powered by TEG is designed. In that work, an intermittent cooling was achieved with an energy harvesting and storage between the TEG and the 
fan, enabling the system cooling for processor overclock. A modeling approach was presented by Martinez et al. [9], who did not provide experimental data on a real prototype.

Many other contributions discuss efficient energy harvesting solutions: for example, several works of Spies et al. [21] and the number of contributions in wearable devices powered by human body heat [20], [10].

In this paper, we present the results of an investigation on the feasibility of a passive-yet-active cooling system self-powered by thermoelectric generators. The base idea is to use a thermoelectric generator in the same stack of the heat-sink, to scavenge the needed energy to power a fan, that boosts the heat dissipation. Although a patent application was already submitted [15] in the early 90's, experimental results that confirm the proposed approach are not yet available.

The paper is organized as follows: thermal and electric model are presented in Section 2, while the achieved results are discussed in Section 3, before the concluding remarks.

\section{System prototype and model}

The thermal resistance, between the silicon die and the ambient, determines the operating temperature of the electronics. The clock speed of microprocessors is usually automatically tuned with the internal temperature, to not exceed a safe operating threshold when the CPU is running. A lower thermal resistance,

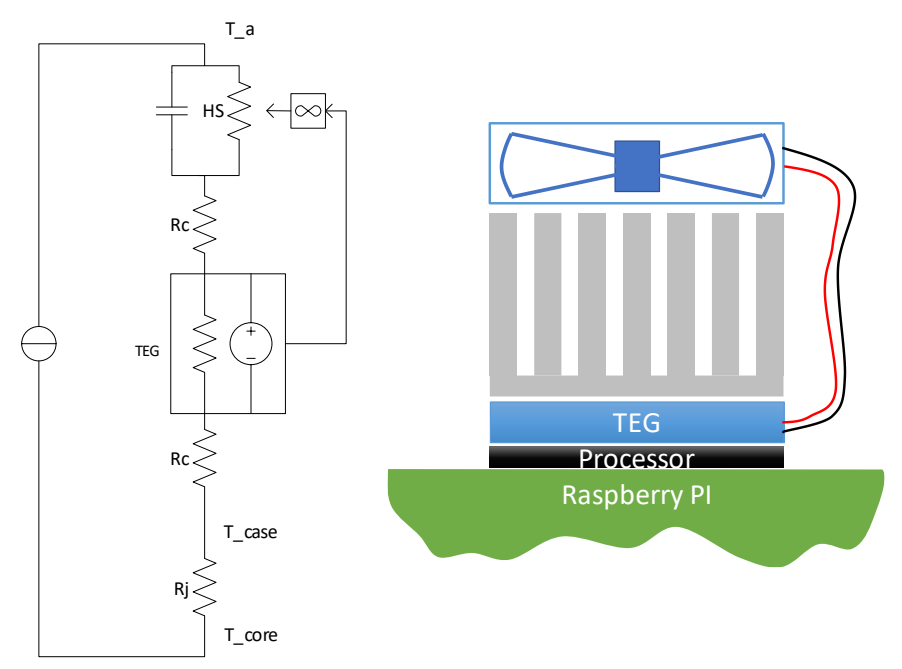

Fig. 1. Thermal model of the thermoelectric energy harvesting cooler and example of the stack implementation. 
therefore, allows the processor to run with faster clock rates, because of the enhanced heat spreading capability. On the other hand, incrementing the airflow through the heat-sink also result in a more efficient heat dissipation. Therefore, even if inserting a TEG between the chip package and the heatsink appears a drawback (because of the higher thermal resistance) the energy produced by this element may be enough to power a fan and then improving the heat-sink dissipation.

The system requires a joint thermal and electrical model to measure the thermal properties and to find the optimal trade-off of the design parameters.

The heat source, used in our experiments, is the microprocessor on a Raspberry PI 3 single board computer (SBC). This was chosen because it is a well known and widely used SBC, although the heat generated by its BCM2837 ARM core is limited compared with other platforms. For instance, a typical Exynos $\mathrm{SoC}$ used for smartphones and tablets can drain 5 to $8 \mathrm{~W}$ under load, while the BCM2837 usually consumes around $3 \mathrm{~W}$, providing, therefore, less heat dissipation usable by the TEG.

The resulting scheme is represented in Fig. 1, both as schematic stack representation, and as the thermal equivalent circuit. Some parameters of the prototype are provided in Table 2 . Because the main purpose of the system is spreading heat off the processor, the energy scavenged by the TEG is reused for boosting the cooling. Therefore the arrow going out of the TEG module in Fig. 1 actually changes the thermal resistance of the heatsink (HS in the figure) by means of a fan (improving the thermal convection on the fins).

In our experiments, we used a Laird CP10-31-05 TEG, and even if the thermal characteristics of the thermoelectric module are not available in the datasheet; we extracted a model from some previous experiments available in literature [7].

The fan is mounted on the top of an aluminum heat-sink of $1.5 \times 1.5 \times 1.5 \mathrm{~cm}$, using a custom 3D-printed support. This support has also the purpose of fos-

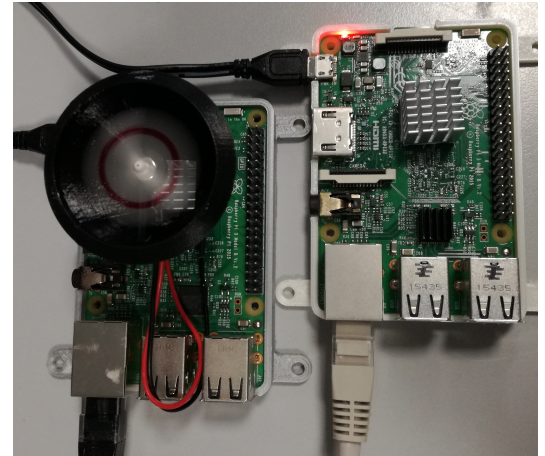

Fig. 2. Two Raspberry PIs, with and without the TEG-powered cooler, used to evaluate the effectiveness of the cooling system running the same benchmark.

\begin{tabular}{cc}
\hline Parameter & Value \\
\hline Fan min Voltage & $80 \mathrm{mV}$ \\
Fan min Power & $1.5 \mathrm{~mW}$ \\
T_core max & $78{ }^{\circ} \mathrm{C}$ \\
ambient Temperature $\sim 25^{\circ} \mathrm{C}$
\end{tabular}

Table 1. Operating parameters of the prototype in the laboratory environment 
tering chimney effect on the heatsink. Fig. 2 shows two Raspberry PIs used for executing a parallel benchmark, with and without the proposed method.

All the parameters identified in the model of the Fig. 1 are estimated and verified by measures. $R c$ is the thermal resistance available both at the interfaces heatsink-to-TEG and TEG-to-processor, and it is realized with a silicone thermal compound, while $R j$ is the thermal resistance at the junction inside the processor case. Both can be considered negligible with respect to the thermal resistance of the TEG and the heat-sink. The junction resistance $R j$ of the BCM2837 is not known actually, but in similar devices, such resistance is as small as $0.5 \mathrm{~K} / \mathrm{W}$.

As widely discussed in the literature, the thermal resistance of a TEG is a function of the current flowing through it [5]. With a parametric test setup, similar to the one presented in [8], the thermal resistance of the TEG has been measured between 5 and $8 \mathrm{~K} / \mathrm{W}$. Similarly, the Thermal resistance of the used aluminum heat-sink is around $11 \mathrm{~K} / \mathrm{W}$, that is a typical value for a small aluminum extruded heat-sink.

\section{Results}

We started a parallel test with two setups, as a first experiment. The main reason for this concurrent experiment is the need of comparable environmental condition, in order to correctly evaluate the performance gain obtained using the TEG and the fan.

In fact, the temperature on the cold side of the TEG (and therefore its power generation) is directly proportional to the amount of heat dissipated by the heat-sink, thus it is important to control the external environmental temperature. With a double test executed in parallel at the same time, and with the

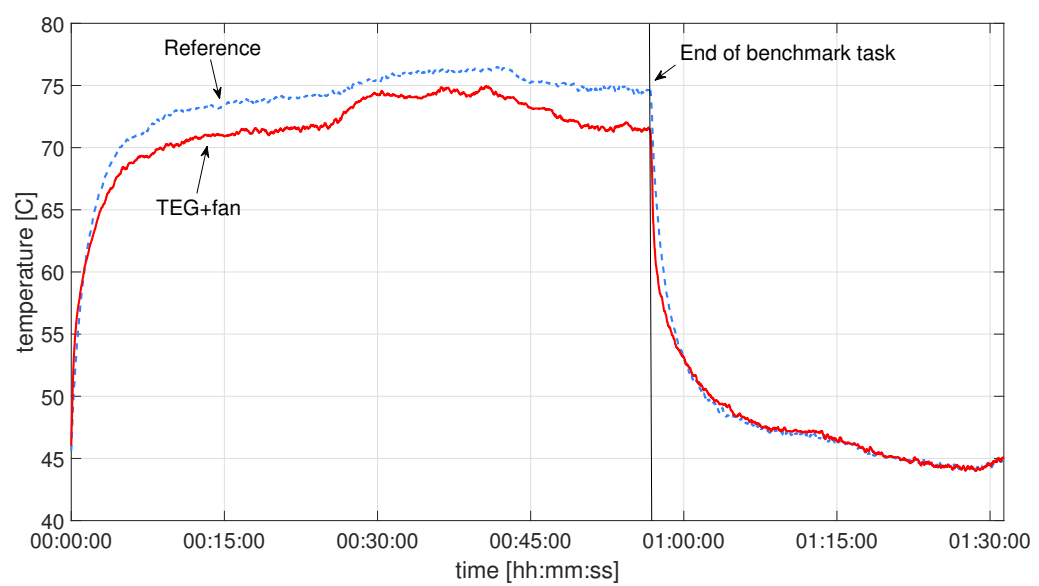

Fig. 3. Resulting temperature on two Raspberry PI processors (T_core) running the same benchmark, at the same time. The reference board has just the heat-sink while the other has the TEG cooler on. 


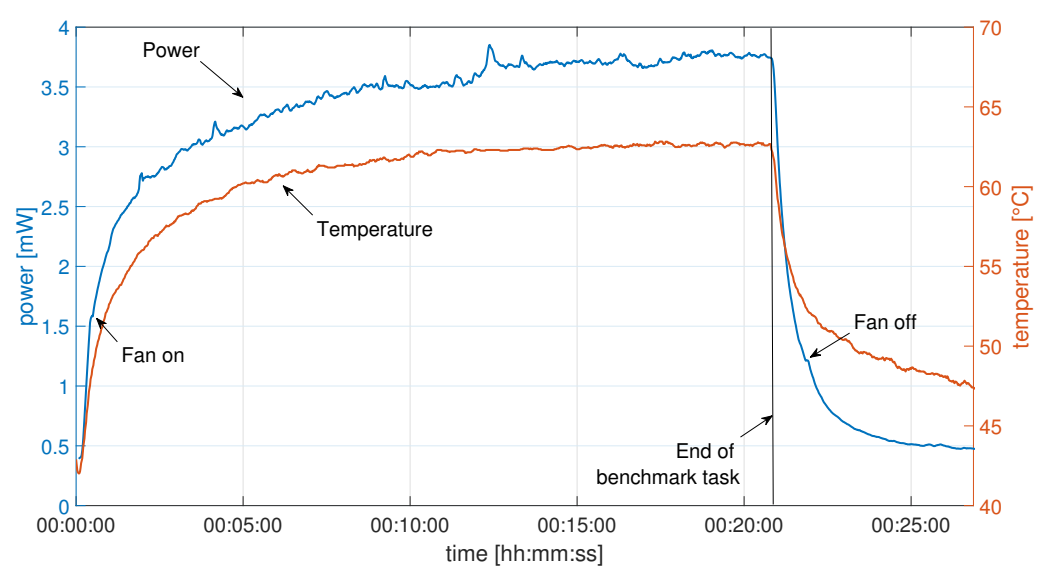

Fig. 4. Power harvested from the TEG and used by the fan, with respect to the temperature on the Raspberry PI core.

same external temperature, we have evaluated the difference generated by the proposed approach.

The temperature on the processor was extracted with a bash script, logging temperature as well as other parameters such as frequencies, voltages, and workload of the CPU. The CPU load was produced with the help of the sysbench tool, commonly available in many Linux distributions. The temperatures logged on the BCM2837 processor are reported in Fig. 3. In the prototype with the TEG cooler, the temperature stabilizes at a couple of degree Celsius lower than the processor with only the heat-sink. The temperature on both the heat-sinks was also logged, finding that, in the case of the TEG-enhanced system, not only the temperature on the processor is lower, but also the heat-sink is kept at lower temperature.

We measured also the power extracted by the TEG (shown in Fig. 3), to assess the possibility of powering the fan indirectly, exploiting a power manager. In fact, the power extracted by the TEG, when the processor is IDLE, is not enough for activating the fan, but it may be stored in an accumulator for later usage, if a power management is implemented.

The advantage would be the recover of all the energy, both during idle and load periods of the processor and the release of the energy on the fan only when needed. We will test this approach in the future, because of some open issues such as the low efficiency of the energy harvester IC in similar conditions, that wastes a remarkable part of the power.

The power generated by the TEG is approximately proportional to the squared of the temperature difference between the device surfaces, which in turn depends on the overall thermal resistance. The latter is decreased by the fan, which works at a higher speed if the power generated is higher, as the chain of dependencies shown as follows: 


$$
P_{T E G} \rightarrow \frac{\text { fan air flow }}{\text { fan power }} \rightarrow \sum R_{\text {thermal }} \rightarrow \Delta T_{T E G} \rightarrow P_{T E G}
$$

The insertion of another relation (the efficiency of an energy harvester, like the LTC3108), between the fan and the power generated by the TEG, lower the overall efficiency of the system, resulting in the loss of the self-sustainability. In fact, the efficiency of the tested energy harvester, in the experiment conditions, is typically lower than $30 \%$. The effect would be an inevitable intermittent operation of the fan. The power harvested by the LTC3108 available at its output (continuously) was about $600 \mu \mathrm{W}$. This is remarkably lower than the one depicted in Fig. 3, which is the energy extracted by the TEG and directly used on the fan.

\section{Conclusion}

In this paper, a prototype of a thermoelectric powered cooler for low-power ARM processor is presented. The system is successfully applied to a Raspberry PI3, showing the advantage of such approach and obtaining a self-powered active cooler capable of decreasing the temperature of some degrees Celsius with respect to a commercial passive cooler.

\section{References}

1. Bergonzini, C., Brunelli, D., Benini, L.: Comparison of energy intake prediction algorithms for systems powered by photovoltaic harvesters. Microelectron. J. 41(11), 766-777 (Nov 2010)

2. Brunelli, D., Dondi, D., Bertacchini, A., Larcher, L., Pavan, P., Benini, L.: Photovoltaic scavenging systems: Modeling and optimization. Microelectronics Journal 40(9), $1337-1344$ (2009)

3. Brunelli, D., Farella, E., Rocchi, L., Dozza, M., Chiari, L., Benini, L.: Bio-feedback system for rehabilitation based on a wireless body area network. In: Fourth Annual IEEE International Conference on Pervasive Computing and Communications Workshops (PERCOMW'06). pp. 5 pp.-531 (March 2006)

4. Brunelli, D., Tosato, P., Rossi, M.: Microbial fuel cell as a biosensor and a power source for flora health monitoring. In: 2016 IEEE SENSORS. pp. 1-3 (Oct 2016)

5. Carmo, J., Antunes, J., Silva, M., Ribeiro, J., Goncalves, L., Correia, J.: Characterization of thermoelectric generators by measuring the load-dependence behavior. Measurement 44(10), 2194-2199 (dec 2011)

6. Champier, D.: Thermoelectric generators: A review of applications. Energy Conversion and Management 140, 167-181 (2017)

7. Dziurdzia, P.: Modeling and simulation of thermoelectric energy harvesting processes. In: Sustainable Energy Harvesting Technologies - Past, Present and Future. InTech (dec 2011)

8. Izidoro, C., Junior, O.A., Carmo, J., Schaeffer, L.: Characterization of thermoelectric generator for energy harvesting. Measurement 106, 283-290 (aug 2017)

9. Martínez, A., Astrain, D., Rodríguez, A.: Dynamic model for simulation of thermoelectric self cooling applications. Energy 55, 1114-1126 (jun 2013) 
10. Nardello, M., Tosato, P., Rossi, M., Brunelli, D.: A thermoelectric powered system for skiing performance monitoring. In: Applications in Electronics Pervading Industry, Environment and Society. pp. 135-144. Springer International Publishing (2019)

11. Pasquato, L., Bonotto, N., Tosato, P., Brunelli, D.: An optimized wind energy harvester for remote pollution monitoring. In: 2017 IEEE Workshop on Environmental, Energy, and Structural Monitoring Systems (EESMS). pp. 1-6 (July 2017)

12. Porcarelli, D., Brunelli, D., Benini, L.: Characterization of lithium-ion capacitors for low-power energy neutral wireless sensor networks. In: 2012 Ninth International Conference on Networked Sensing (INSS). pp. 1-4 (June 2012)

13. Porcarelli, D., Brunelli, D., Benini, L.: Clamp-and-forget: A self-sustainable noninvasive wireless sensor node for smart metering applications. Microelectronics Journal 45(12), 1671 - 1678 (2014)

14. Porcarelli, D., Spenza, D., Brunelli, D., Cammarano, A., Petrioli, C., Benini, L.: Adaptive rectifier driven by power intake predictors for wind energy harvesting sensor networks. IEEE Journal of Emerging and Selected Topics in Power Electronics 3(2), 471-482 (June 2015)

15. Primus, F.J., Goldenberg, M.D., Hills, S.: United States Patent ( 19 ) (1991)

16. Rizzon, L., Rossi, M., Passerone, R., Brunelli, D.: Energy neutral hybrid cooling system for high performance processors. In: International Green Computing Conference. pp. 1-6 (Nov 2014)

17. Rossi, M., Rizzon, L., Fait, M., Passerone, R., Brunelli, D.: Energy neutral wireless sensing for server farms monitoring. IEEE Journal on Emerging and Selected Topics in Circuits and Systems 4(3), 324-334 (Sept 2014)

18. Sartori, D., Brunelli, D.: A smart sensor for precision agriculture powered by microbial fuel cells. In: 2016 IEEE Sensors Applications Symposium (SAS). pp. 1-6 (April 2016)

19. Siddique, A.R.M., Mahmud, S., Heyst, B.V.: A review of the state of the science on wearable thermoelectric power generators (TEGs) and their existing challenges. Renewable and Sustainable Energy Reviews 73(January), 730-744 (2017)

20. Spies, P., Pollak, M., Rohmer, G.: Power management for energy harvesting applications (2018)

21. Zhou, Y., Paul, S., Bhunia, S.: Harvesting Waste Heat in a Microprocessor Using Thermoelectric Generators - Modeling Analysis and Measurement.Pdf pp. 98-103 (2008) 